\title{
Stirling's Approximation for Central Extended Binomial Coefficients
}

\author{
Steffen Eger
}

\begin{abstract}
We derive asymptotic formulas for central extended binomial coefficients, which are generalizations of binomial coefficients. To do so, we relate the exact distribution of the sum of independent discrete uniform random variables to the asymptotic distribution, obtained from the Central Limit Theorem and a local limit variant.
\end{abstract}

\section{Stirling's formula and central binomial coefficients}

For a nonnegative integer $k$, Stirling's formula

$$
k ! \sim \sqrt{2 \pi k}\left(\frac{k}{e}\right)^{k}
$$

where $e$ is Euler's number, yields an approximation of the central binomial coefficient $\left(\begin{array}{c}k \\ k / 2\end{array}\right)$ using $\left(\begin{array}{c}k \\ m\end{array}\right)=\frac{k !}{m !(k-m) !}$ as

$$
\left(\begin{array}{c}
k \\
k / 2
\end{array}\right) \sim \frac{2^{k+1}}{\sqrt{2 \pi k}}
$$

where we write $a_{k} \sim b_{k}$ as short-hand for $\lim _{k \rightarrow \infty} \frac{a_{k}}{b_{k}}=1$. In our current note, we derive asymptotic formulas for central extended binomial, or polynomial, coefficients (cf. [2, 3, 7]). These coefficients appear in the extended binomial triangles (which we also call $(\ell+1)$-nomial, polynomial, or multinomial triangles [8]), which are generalizations of binomial, or Pascal, triangles, in which entries in row $k$ are defined as coefficients of the polynomial $\left(1+x+x^{2}+\cdots+x^{\ell}\right)^{k}$ for $\ell \geq 0$. Our derivation is not based upon asymptotics of factorials, but upon the limiting distribution of the sum of discrete uniform random variables 1

\section{Extended binomial triangles}

In generalization to binomial triangles, $(\ell+1)$-nomial triangles, for $\ell \geq 0$, are defined in the following way. Starting with a 1 in row zero, construct an entry in row $k$, for $k \geq 1$, by adding the overlying $(\ell+1)$ entries in row $(k-1)$ (some of these entries are taken as zero if not defined); thereby, row $k$ has $(k \ell+1)$ entries. For example, the binomial $(\ell=1)$, trinomial $(\ell=2)$, and quadrinomial triangles $(\ell=3)$ start as follows,

\footnotetext{
${ }^{1}$ Throughout, we assume that all fractional values such as $x=\frac{k \ell}{2}$ are integral when used in the context of extended binomial coefficients. If this is not the case, then replace respective quantities with their floor, $\lfloor x\rfloor$, the largest integer less than or equal to $x$.
} 


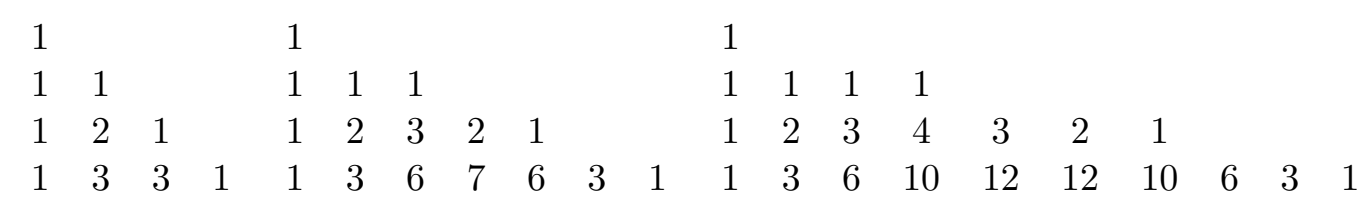

In the $(\ell+1)$-nomial triangle, the $n$th entry, for $0 \leq n \leq k \ell$ in row $k$, which we denote by $\left(\begin{array}{l}k \\ n\end{array}\right)_{\ell+1}$, has the following interpretation. It is the coefficient of $x^{n}$ in the expansion of

$$
\left(1+x+x^{2}+\cdots+x^{\ell}\right)^{k}=\sum_{n=0}^{k \ell}\left(\begin{array}{l}
k \\
n
\end{array}\right)_{\ell+1} x^{n} .
$$

It has been shown that $\left(\begin{array}{l}k \\ n\end{array}\right)_{\ell+1}$ denotes the number of restricted integer compositions (for a definition, see, e.g., [9] and many others) of the nonnegative integer $n$ with $k$ parts $\pi_{1}, \ldots, \pi_{k}$, each from the set $\{0,1, \ldots, \ell\}$ (cf. [5]), and allows the following representation,

$$
\left(\begin{array}{l}
k \\
n
\end{array}\right)_{\ell+1}=\sum_{\substack{k_{0} \geq 0, \ldots, k_{\ell} \geq 0 \\
k_{0}+\cdots+k_{\ell}=k \\
0 \cdot k_{0}+1 \cdot k_{1}+\cdots+\ell \cdot k_{\ell}=n}}\left(\begin{array}{c}
k \\
k_{0}, \ldots, k_{\ell}
\end{array}\right)
$$

where $\left(\begin{array}{c}k \\ k_{0}, \ldots, k_{\ell}\end{array}\right)$ is a multinomial coefficient, defined as $\frac{k !}{k_{0} ! \cdots k_{\ell} !}$, for nonnegative integers $k_{0}, \ldots, k_{\ell}$. We can verify representation (2) by noting that for real numbers $x_{0}, \ldots, x_{\ell}$, the multinomial theorem (cf. [15]) states that

$$
\left(x_{0}+x_{1}+\cdots+x_{\ell}\right)^{k}=\sum_{\substack{k_{0} \geq 0, \ldots, k_{\ell} \geq 0 \\
k_{0}+\cdots+k_{\ell}=k}}\left(\begin{array}{c}
k \\
k_{0}, \ldots, k_{\ell}
\end{array}\right) x_{0}^{k_{0}} \cdots x_{\ell}^{k_{\ell}} .
$$

Thus, setting $x_{i}=x^{i}$ for $i=0, \ldots, \ell$,

$$
\left(1+x^{1}+\cdots+x^{\ell}\right)^{k}=\sum_{\substack{k_{0} \geq 0, \ldots, k_{\ell} \geq 0 \\
k_{0}+\cdots+k_{\ell}=k}}\left(\begin{array}{c}
k \\
k_{0}, \ldots, k_{\ell}
\end{array}\right) x^{0 \cdot k_{0}+\cdots+\ell \cdot k_{\ell}}
$$

so that comparing coefficients of the right-hand sides of (11) and (3) leads to (2).

\section{Generalized Stirling's approximation}

Our strategy for deriving approximation formulas for central extended binomial coefficients is as follows. First, we determine the asymptotic distribution of the sum of discrete uniform variables, which we easily find to be a normal distribution by the Central Limit Theorem (CLT). Then, we determine the exact distribution, which turns out to yield the normalized extended binomial coefficients $\left(\begin{array}{l}k \\ n\end{array}\right)_{\ell+1}$. By relating the density of the asymptotic distribution to the density of the exact distribution (e.g., via a 'local limit' argument), we obtain an extended binomial analgoue of Stirling's approximation to central binomial coefficients. 


\subsection{Step 1: Asymptotic distribution of the sum of discrete uniform variables}

Let $k$ be a positive integer and let $\ell$ be a nonnegative integer. Let $X_{j}$, for $j=1, \ldots, k$, be identically and independently distributed random draws from the discrete uniform distribution on the set $\{0, \ldots, \ell\}$, and let $S_{k}$ be their sum,

$$
S_{k}=\sum_{j=1}^{k} X_{j}
$$

By standard moments of the uniform distribution, the mean and variance of each $X_{j}$ are given by

$$
\mu=\mathrm{E}\left[X_{j}\right]=\frac{\ell}{2}, \quad \text { and } \quad \sigma^{2}=\operatorname{Var}\left[X_{j}\right]=\frac{(\ell+1)^{2}-1}{12} .
$$

Hence, by independent and identical distribution of $X_{1}, \ldots, X_{k}$, and application of the CLT, the random variable $\sqrt{k}\left(\frac{S_{k}}{k}-\mu\right)$ converges, as $k \rightarrow \infty$, in distribution to a normal $\mathcal{N}\left(0, \sigma^{2}\right)$ distributed random variable. Recall that convergence in distribution precisely means that the cumulative density function of $\sqrt{k}\left(\frac{S_{k}}{k}-\mu\right)$ converges pointwise to the cumulative density function of the $\mathcal{N}\left(0, \sigma^{2}\right)$ distribution.

\subsection{Step 2: Exact distribution of the sum of discrete uniform random variables}

We now determine exactly the probability that $S_{k}$ takes on the integer value $n$, for $0 \leq n \leq k \ell$. To do so, we consider 'isomorphic copies' $\tilde{X}_{j}$ of $X_{j}$, which are independently and identically multinomially distributed with probabilities $p_{0}=\cdots=p_{\ell}=\frac{1}{\ell+1}$ of types 0 to $\ell$. Each $\tilde{X}_{j}=\left(A_{0}, \ldots, A_{\ell}\right)$ is vectorvalued, with $P\left[\tilde{X}_{j}=\left(a_{0}, \ldots, a_{\ell}\right)\right]=\frac{1}{\ell+1}$ for nonnegative integers $a_{s}$, with $a_{0}+\cdots+a_{\ell}=1$, where $A_{s}$ denotes the number of times an event of type $s$, for $s=0, \ldots, \ell$, occurs. Then, the sum $\tilde{S}_{k}=\tilde{X}_{1}+\cdots+\tilde{X}_{k}$ has the interpretation of representing the event of drawing with replacement $k$ balls of $(\ell+1)$ different types from a bag, where the probability of drawing type $s=0, \ldots, \ell$ is $\frac{1}{\ell+1}$. Thus, by the standard interpretation of the multinomial distribution, $\tilde{S}_{k}$ has density

$$
P\left[\tilde{S}_{k}=\left(a_{0}, \ldots, a_{\ell}\right)\right]=P\left[A_{0}=a_{0}, \ldots, A_{\ell}=a_{\ell}\right]=\left(\begin{array}{c}
k \\
a_{0}, \ldots, a_{\ell}
\end{array}\right)\left(\frac{1}{\ell+1}\right)^{k},
$$

where $a_{0}+\cdots+a_{\ell}=k$ for nonnegative integers $a_{0}, \ldots, a_{\ell}$. Then, if $\tilde{S}_{k}=\left(a_{0}, \ldots, a_{\ell}\right), S_{k}$, the variable corresponding to $\tilde{S}_{k}$, represents the integer $0 \cdot a_{0}+\cdots+\ell \cdot a_{\ell}$. Thus, for $n$ such that $0 \leq n \leq k \ell$,

$$
P\left[S_{k}=n\right]=\sum_{\substack{a_{0} \geq 0, \ldots, a_{\ell} \geq 0 \\
a_{0}+\cdots+a_{\ell}=k \\
0 \cdot a_{0}+\cdots+\ell \cdot a_{\ell}=n}} P\left[\tilde{S}_{k}=\left(a_{0}, \ldots, a_{\ell}\right)\right]=\left(\frac{1}{\ell+1}\right)^{k}\left(\begin{array}{l}
k \\
n
\end{array}\right)_{\ell+1},
$$

using representation (2).

An arguably more straightfoward derivation of the exact distribution of $S_{k}$, making use of probability generating functions (pgfs), can be given by noting that the pgf $G_{X_{j}}(x)=\sum_{n \geq 0} P\left[X_{j}=\right.$ $n] x^{n}$ of each $X_{j}$ is given by

$$
G_{X_{j}}(x)=\frac{1}{\ell+1} \sum_{n=0}^{\ell} x^{n}
$$


By independence of $X_{1}, \ldots, X_{k}$, the pgf of $S_{k}$ is hence given as,

$$
G_{S_{k}}(x)=G_{X_{1}}(x) \cdots G_{X_{k}}(x)=\left(\frac{1}{\ell+1}\right)^{k}\left(\sum_{n=0}^{\ell} x^{n}\right)^{k}=\left(\frac{1}{\ell+1}\right)^{k} \sum_{n=0}^{k \ell}\left(\begin{array}{l}
k \\
n
\end{array}\right)_{\ell+1} x^{n} .
$$

Thus,

$$
P\left[S_{k}=n\right]=\frac{G_{S_{k}}^{(n)}(0)}{n !}=\left(\frac{1}{\ell+1}\right)^{k} \frac{n !}{n !}\left(\begin{array}{l}
k \\
n
\end{array}\right)_{\ell+1}=\left(\frac{1}{\ell+1}\right)^{k}\left(\begin{array}{l}
k \\
n
\end{array}\right)_{\ell+1},
$$

where we denote by $G_{X}^{(n)}(0)$ the $n$th derivative of $G_{X}$, evaluated at zero.

\subsection{Step 3: Local limit theorem}

To derive an asymptotic formula for $\left(\begin{array}{l}k \\ n\end{array}\right)_{\ell+1}$, we would like to make use of the results derived in Steps 1 and 2 above. Ideally, we would like to equate the probability density function of the asymptotic normal dstribution of $S_{k}$ with the exact distribution. However, as mentioned, convergence in distribution, as assured by the CLT, only guarantees pointwise convergence of cumulative density functions. On the contrary, 'local limit theorems' describe how the probability density function of a sum of random variables approaches the normal density function. For integer-valued random variables (also called lattice or arithmetical distributions), Gnedenko and Kolmogorov [10] provide the following result.

Theorem 3.1. If $X_{1}, X_{2}, \ldots$ are independent lattice random variables with identical distribution with finite mean $\mu$ and variance $\sigma^{2}$, such that the greatest common divisor of the differences of all the values of $X_{j}$ taken with positive probability is 1 , then

$$
\left|\sqrt{k} \sigma P\left[S_{k}=n\right]-\frac{1}{\sqrt{2 \pi}} e^{-\frac{(n-k \mu)^{2}}{2 \sigma^{2} k}}\right| \rightarrow 0
$$

uniformly in $n$ as $k \rightarrow \infty$.

Since in our situation, the set of values of each $X_{j}$ taken with positive probability is $\{0, \ldots, \ell\}$, the greatest common divisor of the differences is clearly 1 . Thus, all assumptions of Theorem 3.1 are satisfied in our case, and, hence, also its consequences hold. Therefore, the following approximation is suggested for large $k$ :

$$
\sqrt{k} \sigma P\left[S_{k}=n\right] \sim \frac{1}{\sqrt{2 \pi}} e^{-\frac{(n-k \mu)^{2}}{2 \sigma^{2} k}} .
$$

For $n=k \mu=k \ell / 2$, the argument to the exponential function is zero, and thus

$$
\sqrt{k} \sigma P\left[S_{k}=k \ell / 2\right] \sim \frac{1}{\sqrt{2 \pi}}, \quad \text { or equivalently, } \quad P\left[S_{k}=k \ell / 2\right] \sim \frac{1}{\sqrt{2 \pi \sigma^{2} k}} .
$$

Using the exact form for $P\left[S_{k}=n\right]$ from Step 2 above, we hence have, bringing the normalizing term $(\ell+1)^{k}$ to the right-hand side,

$$
\left(\begin{array}{c}
k \\
\frac{k \ell}{2}
\end{array}\right)_{\ell+1} \sim \frac{(\ell+1)^{k}}{\sqrt{2 \pi k \frac{(\ell+1)^{2}-1}{12}}} .
$$


For example, for $\ell=1$, Pascal's case, $\ell=2, \ell=3$, and $\ell=4$, we therefore have the approximations

$$
\left(\begin{array}{c}
k \\
\frac{k}{2}
\end{array}\right) \sim \frac{2^{k+1}}{\sqrt{2 \pi k}}, \quad\left(\begin{array}{l}
k \\
k
\end{array}\right)_{3} \sim \frac{3^{k}}{\sqrt{\frac{4}{3} \pi k}}, \quad\left(\begin{array}{c}
k \\
\frac{3}{2} k
\end{array}\right)_{4} \sim \frac{4^{k}}{\sqrt{\frac{5}{2} \pi k}}, \quad \text { and } \quad\left(\begin{array}{c}
k \\
2 k
\end{array}\right)_{5} \sim \frac{5^{k}}{2 \sqrt{\pi k}} .
$$

In Figure 1, we show for $\ell=4$ the distributions $P\left[S_{k}=n\right]$ for $k=5,10,20$, and their respective normal approximations. There, we can see the local limit theorem 'at work': The exact density function apparently approaches, pointwise, the normal density function.
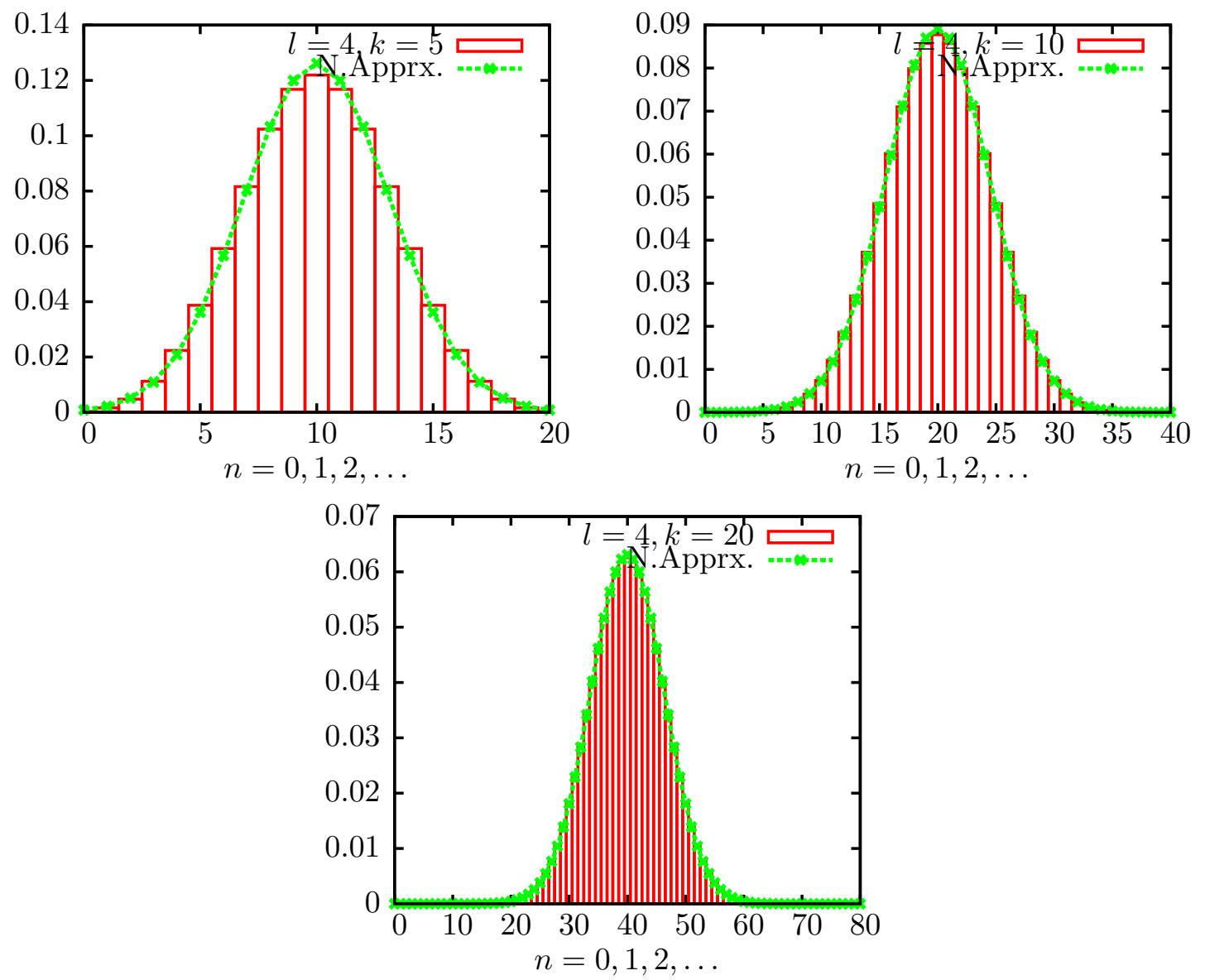

Figure 1: Distributions $P\left[S_{k}=n\right]$ for $k=5,10,20$, for $l=4$ fixed, and normal approximations.

\section{Discussion}

Although extended binomial coefficients, together with their connection to the sum of discrete uniform random variables, go back at least to De Moivre's Doctrine of Chances [4] and to Euler's [6] analytical study of the coefficients of polynomial (11), the mathematics community has apparently more or less ignored their systematic study, except for a few recent publications such as [1, 2, 5, 7, 8]. Next, using the CLT (or a local limit variant) to deduce asymptotics of mathematical objects has 
been suggested, for example, by Walsh [14], who derives Stirling's formula for factorials by equating the distribution of the sum of Poisson distributed random variables with the normal density. Finally, the asymptotics of both the central binomial $(\ell=1)$ as well as the central trinomial coefficients $(\ell=2)$ seem to be known (e.g. [7, 12]), while the general formula (5) is, to the best of our knowledge, novel. However, Ratsaby [13] derives our general result (4), as an estimate of the number of restricted integer compositions, by application of Cauchy's coefficient formula to the polynomial (1) and computation of the resulting integral by Laplace's method for evaluation of integrals. A historical perspective of local versus central limit theorem is provided by McDonald [11.

\section{References}

[1] Richard C. Bollinger and Charles L. Burchard. Lucas's theorem and some related results for extended pascal triangles. American Mathematical Monthly, 97(3):198-204, 1990.

[2] C.C.S. Caiado and P.N. Rathie. Polynomial coefficients and distribution of the sum of discrete uniform variables. In A. M. Mathai, M. A. Pathan, K. K. Jose, and Joy Jacob, editors, Eighth Annual Conference of the Society of Special Functions and their Applications. Society for Special Functions \& their Applications, January 2007.

[3] Louis Comtet. Advanced combinatorics: the art of finite and infinite expansions. Reidel, Dordrecht, 1974.

[4] Abraham DeMoivre. The Doctrine of Chances: Or, A Method of Calculating the Probabilities of Events in Play. Millar, Chelsea, New York, 1967, 3rd edition, 1756. Reprint of the third (1756) edition.

[5] Steffen Eger. Restricted weighted integer compositions and extended binomial coefficients. Journal of Integer Sequences [electronic only], 16(1):Article 13.1.3, 25 p., electronic only, 2013.

[6] Leonhard Euler. De evolutione potestatis polynomialis cuiuscunque $\left(1+x+x^{2}+\cdots\right)^{n}$. Nova Acta Academiae Scientarum Imperialis Petropolitinae, 12, 1801.

[7] N.-E. Fahssi. The polynomial triangles revisited, 2012. available at http://arxiv.org/abs/1202.0228.

[8] Daniel C. Fielder and Cecil O. Alford. Pascal's Triangle: Top Gun or Just One of the Gang?, pages 77-90. Springer Netherlands, Dordrecht, 1991.

[9] Philippe Flajolet and Robert Sedgewick. Analytic Combinatorics. Cambridge University Press, New York, NY, USA, 1 edition, 2009.

[10] B. V. Gnedenko and A. N. Kolmogorov. Limit distributions for sums of independent random variables. Translated from the Russian, annotated, and revised by K. L. Chung. With appendices by J. L. Doob and P. L. Hsu. Revised edition. Addison-Wesley Publishing Co., Reading, Mass.-London-Don Mills., Ont., 1968.

[11] D.R. McDonald. The local limit theorem: A historical perspective. JIRSS, 4:73-86, 2005. 
[12] The On-Line Encyclopedia of Integer Sequences. available at http://oeis.org, 2012, Sequence A002426.

[13] Joel Ratsaby. Estimate of the number of restricted integer-partitions. Applicable Analysis and Discrete Mathematics, 2(2):222-233, 2008.

[14] D.P. Walsh. Equating Poisson and normal probability functions to derive Stirling's formula. American Statistician, 49:270-271, 1995.

[15] Eric Weisstein. Multinomial Series — From MathWorld, A Wolfram Web Resource, available at http://mathworld.wolfram.com/MultinomialSeries.html. 